\title{
As borboletas (Lepidoptera, Papilionoidea) do Distrito Federal, Brasil
}

\author{
Eduardo de Oliveira Emery ${ }^{1}$, Keith S. Brown $\mathrm{Jr}^{2}$ \& Carlos E. G. Pinheiro ${ }^{3}$
}

${ }^{1}$ Departamento de Zoologia, Instituto de Biologia, Universidade de Brasília, UnB-Programa de pós-graduação em Biologia Animal, 70910-900 Brasília-DF, Brasil. eoemery@unb.br

${ }^{2}$ Museu de História Natural, Universidade Estadual de Campinas-Unicamp. Caixa Postal 6109, 13084-971 Campinas-SP, Brasil. ksbrown@unicamp.br

${ }^{3}$ Departamento de Zoologia, Instituto de Biologia, Universidade de Brasília-UnB, 70910-900 Brasília-DF, Brasil. cegp@unb.br

\begin{abstract}
The butterflies (Lepidoptera, Papilionoidea) of the Distrito Federal, Brazil. An updated list containing 504 species and 506 subspecies of butterflies (Papilionoidea) found in the Distrito Federal (central Brazil) in the last four decades is presented in this study, including data obtained in the literature, our personal collections, and several entomological collections recently visited, but not species with presumed distribution in the region. Some aspects concerning the occurrence of threatened species and the conservation of the butterfly fauna in the Distrito Federal are also discussed.
\end{abstract}

KEYWORDS. Central Brazil; cerrado vegetation; conservation; diversity; Neotropical.

RESUMO. As borboletas (Lepidoptera, Papilionoidea) do Distrito Federal, Brasil. Neste estudo é apresentada uma listagem atualizada com 504 espécies e 506 subespécies de borboletas (Papilionoidea) observadas no Distrito Federal nas últimas quatro décadas, onde foram reunidos dados da bibliografia, de coletas pessoais e de várias coleções entomológicas; espécies com ocorrência apenas presumida não foram incluídas. Alguns aspectos relacionados à ocorrência de espécies ameaçadas e à conservação da fauna de borboletas no Distrito Federal são também discutidos.

PALAVRAS-CHAVE. Brasil central; cerrado; conservação; diversidade; Neotropical.

Situado na porção central do Planalto Central Brasileiro (15 $30^{\prime}-16^{\circ} 03^{\prime} \mathrm{W}$ e $47^{\circ} 18^{\prime}-48^{\circ} 17^{\prime} \mathrm{S}$; 750-1350 m altitude) o Distrito Federal ocupa uma área de aproximadamente 5.800 $\mathrm{km}^{2}$. O clima da região é caracterizado por uma estação quente e chuvosa, de outubro a abril e outra fria e seca, de maio a setembro, com temperatura média anual variando entre $18 \mathrm{e}$ $22^{\circ} \mathrm{C}$ e precipitação média anual de $1600 \mathrm{~mm}$. Nesta região encontra-se uma grande diversidade de fisionomias de vegetação de cerrado, entre as quais podemos citar o cerrado sensu strictu, campos cerrados, cerradões, veredas, matas de galeria e extensões de florestas semidecíduas, também conhecidas como florestas mesofíticas, encontradas sobre os afloramentos de solos calcários ou nas baixadas próximas aos rios (Goodland 1971; Eiten 1972). Estas paisagens bastante heterogêneas se constituem nos habitats preferidos de uma enorme variedade de borboletas que aí encontram as condições necessárias para sua sobrevivência.

O primeiro estudo aprofundado sobre a fauna de borboletas do cerrado foi realizado por Brown \& Mielke (1967a, b) que apresentaram uma listagem com aproximadamente 700 espécies de borboletas (Papilionoidea e Hesperioidea) em toda a região do cerrado, muitas delas encontradas no Distrito Federal. Estes autores também analisaram as relações faunísticas do cerrado com outros biomas brasileiros, indicando que a fauna do cerrado tem muitas espécies ou subespécies em comum com a mata atlântica (veja também Brown \& Freitas 2000), a floresta amazônica, a caatinga e outros biomas que o cercam, além de um número relativamente grande de espécies com ampla distribuição geográfica. O número de espécies ou subespécies endêmicas do cerrado é pequeno $(<6 \%)$.
Nestes últimos quarenta anos vários outros estudos envolvendo levantamentos da fauna de borboletas foram realizados no Distrito Federal (Pinheiro \& Ortiz 1992; Diniz \& Morais 1995, 1997). Neste período novas espécies e subespécies foram descritas e novas classificações envolvendo os diversos taxa de borboletas foram também propostas (Brown 1992; Tyler et al. 1994; Lamas 2004). Além disso, o Distrito Federal vem passando por um intenso processo de urbanização, pela implantação de atividades agrícolas e outras atividades econômicas que levam inexoravelmente à destruição do habitat natural. A necessidade de uma lista atualizada de espécies é, portanto, fundamental para a avaliação e o monitoramento da perda em biodiversidade de borboletas, bem como para o desenvolvimento de diferentes tipos de pesquisas envolvendo estes insetos.

Neste estudo foram reunidos dados da bibliografia, de coletas pessoais e outros compilados em coleções entomológicas para produzir uma listagem atualizada das espécies de borboletas (Papilionoidea) do Distrito Federal. Alguns aspectos relacionados à ocorrência de espécies ameaçadas e à conservação da fauna de borboletas são também discutidos.

\section{MATERIAL E MÉTODOS}

A listagem de espécies apresentada a seguir foi baseada nos levantamentos de fauna realizados por Brown \& Mielke $(1967 a, b)$ nas florestas de Sobradinho e matas de galeria do Rio Sobradinho, Chapada da Contagem, região da Fercal, Brasília Country Clube, Jardim Zoológico de Brasília, Reserva 
Ecológica do Gama e Rio Maranhão, por Pinheiro \& Ortiz (1992) na Estação Ecológica do Jardim Botânico, por Pinheiro et al. (1992) no Parque Nacional de Brasília, por Diniz \& Morais $(1995,1997)$ na Fazenda Água Limpa (UnB), Reserva Ecológica do IBGE e Parque Nacional de Brasília, por Pinheiro \& Emery (dados não pub.) na Estação Ecológica de Águas Emendadas, Fazenda Água Limpa, Reserva do Centro Olímpico (campus da UnB), Poço Azul, Vila Telebrasília, Reserva do IBGE, Reserva do CPAC (Embrapa) e em várias localidades na bacia do Rio Maranhão, além de dados obtidos nas coleções entomológicas da Universidade de Brasília, do IBGE, do Centro de Pesquisas Agropecuária do Cerrado (EMBRAPA) e da Coleção de Entomologia Pe. J. S. Moure, Departamento de Zoologia, Universidade Federal do Paraná. Espécies com distribuições geográficas presumidas ou encontradas apenas em regiões vizinhas ao Distrito Federal não foram incluídas.

A classificação adotada segue Tyler et al. (1994) para os Papilionidae e Lamas (2004) para os demais grupos de Papilionoidea.

\section{RESULTADOS E DISCUSSÃO}

Um total de 504 espécies e 506 subespécies de Papilionoidea foi registrado no Distrito Federal. A classificação adotada e o número de espécies encontrado em cada grupo taxonômico estão apresentados na Tabela I. A lista atualizada de espécies é apresentada na Tabela II. Este grande número de Papilionoidea demonstra claramente a grande riqueza de espécies desta região dos cerrados brasileiros. Entretanto, podemos esperar que o número de espécies que efetivamente ocorrem no Distrito Federal seja ainda maior, pois algumas regiões ainda não foram satisfatoriamente amostradas. Brown \& Freitas (2000) estimam que o número de borboletas (Papilionoidea e Hesperioideia) no "Brasil central" deve chegar a 1.000. Podemos ainda esperar que novas espécies sejam adicionadas a esta lista com o avanço das pesquisas em taxonomia, especialmente nas famílias Hesperiidae, Lycaenidae, Riodinidae e Nymphalidae (Satyrinae).

Poucas espécies consideradas vulneráveis ou ameaçadas de extinção estão presentes nesta listagem (Tabela II). Entre elas merecem atenção especial: Parides burchellanus (Papilionidae, Troidini), que consta na lista de espécies ameaçadas da IUCN Red Data Book (Collins \& Morris 1985), Magnastigma julia (Lycaenidae, Theclinae) e Agrias claudina godmani (Nymphalidae, Charaxinae), que constam do catálogo de espécies ameaçadas do Estado de Minas Gerais (Brown, Mielke \& Casagrande 1988). Populações isoladas de Parides burchellanus e Agrias claudina godmani foram recentemente encontradas em diferentes locais do Distrito Federal. Populações de Magnastigma julia foram observadas na Estação Ecológica do Jardim Botânico, na Fazenda Água Limpa e na Reserva do IBGE.

O grande risco a que toda a fauna local de borboletas está exposta decorre da destruição e fragmentação dos seus habitats naturais. Cerca de 150 espécies desta fauna ainda não foi registrada em qualquer unidade de conservação do
Tabela I. Classificação das borboletas (Papilionoidea) do Distrito Federal e o número de espécies e subespécies encontradas em cada grupo taxonômico.

\begin{tabular}{|c|c|c|c|c|}
\hline Família & Subfamília & Tribo & $\begin{array}{c}\mathrm{N}^{\mathrm{o}} \mathrm{de} \\
\text { espécies }\end{array}$ & $\begin{array}{r}\mathrm{N}^{\mathrm{o}} \mathrm{de} \\
\text { subespécies }\end{array}$ \\
\hline \multirow[t]{3}{*}{ Papilionidae } & Papilioninae & Graphiini & 4 & 4 \\
\hline & & Troidini & 6 & 7 \\
\hline & & Papilionini & 6 & 6 \\
\hline \multirow[t]{4}{*}{ Pieridae } & Dismorphiinae & & 4 & 4 \\
\hline & Coliadinae & & 16 & 16 \\
\hline & Pierinae & Anthocharidini & 2 & 2 \\
\hline & & Pierini & 4 & 4 \\
\hline \multirow[t]{2}{*}{ Lycaenidae } & Polyommatinae & & 4 & 4 \\
\hline & Theclinae & Eumaeini & 108 & 108 \\
\hline \multirow[t]{9}{*}{ Riodinidae } & Euselasiinae & Euselasiini & 7 & 7 \\
\hline & Riodininae & Mesosemiini & 16 & 16 \\
\hline & & Eurybiini & 4 & 4 \\
\hline & & Riodinini & 42 & 42 \\
\hline & & Symachiini & 14 & 14 \\
\hline & & Helicopini & 4 & 4 \\
\hline & & Incertae sedis & 11 & 11 \\
\hline & & Nymphidiini & 37 & 37 \\
\hline & & Stalachtini & 2 & 2 \\
\hline \multirow[t]{26}{*}{ Nymphalidae } & e Libytheinae & & 1 & 1 \\
\hline & Danainae & Danaini & 4 & 4 \\
\hline & Ithomiinae & Tithoreini & 3 & 3 \\
\hline & & Mechanitini & 4 & 4 \\
\hline & & Napeogenini & 2 & 2 \\
\hline & & Ithomiini & 2 & 2 \\
\hline & & Oleriini & 1 & 1 \\
\hline & & Dircennini & 3 & 3 \\
\hline & & Godyridini & 8 & 8 \\
\hline & Morphinae & Morphini & 5 & 5 \\
\hline & & Brassolini & 18 & 19 \\
\hline & Satyrinae & Satyrini & 46 & 46 \\
\hline & & Incertae sedis & 2 & 2 \\
\hline & Charaxinae & Anaeini & 7 & 7 \\
\hline & & Preponini & 8 & 8 \\
\hline & Biblidinae & Cyrestini & 2 & 2 \\
\hline & & Biblidini & 39 & 39 \\
\hline & Apaturinae & & 3 & 3 \\
\hline & Nymphalinae & Coenini & 5 & 5 \\
\hline & & Nymphalini & 3 & 3 \\
\hline & & Kallimini & 5 & 5 \\
\hline & & Melitaenini & 9 & 9 \\
\hline & Limenitidinae & Limenitidini & 13 & 13 \\
\hline & Heliconiinae & Argynnini & 1 & 1 \\
\hline & & Acraeini & 8 & 8 \\
\hline & & Heliconiini & 11 & 11 \\
\hline \multicolumn{3}{|l|}{ Total } & 504 & 506 \\
\hline
\end{tabular}

Distrito Federal, sendo que a maior parte destas espécies está concentrada nas florestas semidecíduas que ocorrem sobre solos calcários nas regiões de Sobradinho, Chapada da Contagem e Fercal, ou em matas de galeria associadas aos rios de médio e grande porte, dois ambientes que não estão representados nas reservas, estações e parques aí encontrados (Pinheiro 2006).

$\mathrm{O}$ fato de ocorrer em áreas protegidas, por si só, também 
Tabela II. Lista de borboletas (Papilionoidea) do Distrito Federal.

PAPILIONOIDEA PAPILIONIDAE

Papilioninae - Graphiini

$1 \quad$ Protesilaus aguiari (d'Almeida, 1937)

$2 \quad$ Protesilaus protesilaus (Linnaeus, 1758)

3 Protesilaus telesilaus telesilaus (C. Felder \& R. Felder, 1864)

$4 \quad$ Mimoides pausanias (Hewitson, 1852)

Papilioninae - Troidini

$1 \quad$ Battus crassus crassus (Cramer, 1777)

2 Battus polydamas polydamas (Linnaeus, 1758)

3 Parides anchises foetterlei (Rothschild \& Jordan, 1906)

$4 \quad$ Parides anchises nephalion (Godart, 1819)

$5 \quad$ Parides bunichus diodorus (Hopffer, 1865)

$6 \quad$ Parides burchellanus (Westwood, 1872)

$7 \quad$ Parides neophilus eurybates (Gray, [1853])

Papilioninae - Papilionini

$1 \quad$ Heraclides anchisiades capys (Hübner, [1809])

$2 \quad$ Heraclides astyalus astyalus (Godart, 1819)

$3 \quad$ Heraclides hectorides (Esper, 1794)

$4 \quad$ Heraclides thoas brasiliensis (Rothschild \& Jordan, 1906)

$5 \quad$ Heraclides torquatus polybius (Swainson, 1823)

$6 \quad$ Pterourus scamander grayi (Boisduval, 1836)

PIERIDAE

Dismorphiinae

1 Dismorphia amphione astynome (Dalman, 1823)

2 Dismorphia thermesia thermesia (Godart, 1819)

3 Enantia lina psamathe (Fabricius, 1793)

$4 \quad$ Pseudopieris nehemia nehemia (Boisduval, 1836)

Coliadinae

Anteos clorinde (Godart, [1824])

Anteos menippe (Hübner, [1818])

Phoebis argante argante (Fabricius, 1775)

Phoebis neocypris neocypris (Hübner, [1823])

Phoebis philea philea (Linnaeus, 1763)

Phoebis sennae sennae (Linnaeus, 1758)

Rhabdodryas trite banksi (Breyer, 1939)

Aphrissa statira statira (Cramer, 1777)

Pyrisitia leuce leuce (Boisduval, 1836)

Pyrisitia nise tenella (Boisduval, 1836)

Eurema albula albula (Cramer, 1775)

Eurema agave pallida (Chavannes, 1850)

Eurema arbela arbela Geyer, 1832

Eurema deva doris (Röber, 1909)

Eurema elathea elathea (Cramer, 1777)

Eurema phiale paula (Röber, 1909)

Pierinae - Anthocharidini

$1 \quad$ Hesperocharis anguitea anguitea (Godart, 1819)

2 Cunizza hirlanda planasia Fruhstorfer, 1910

Pierinae - Pierini

1 Archonias brassolis tereas (Godart, 1819)

2 Melete lycimnia paulista Fruhstorfer, 1908

3 Appias drusilla drusilla (Cramer, 1777)

$4 \quad$ Ascia monuste orseis (Godart, 1819)
Tabela II Continuação.

\section{LYCAENIDAE}

Polyommatinae

Leptotes cassius cassius (Cramer, 1775)

Hemiargus hanno (Stoll, 1790)

Zizula cyna (Edwards, 1881)

Elkalyce cogina (Schaus, 1902)

Theclinae - Eumaeini

Paiwarria aphaca (Hewitson, 1867)

Paiwarria venulius (Cramer, 1779)

Evenus regalis (Cramer, 1775)

Evenus satyroides (Hewitson, 1865)

Arcas imperialis (Cramer, 1775)

Atlides rustan (Stoll, 1790)

Pseudolycaena marsyas (Linnaeus, 1758)

Theritas triquetra (Hewitson, 1865)

Theritas hemon (Cramer, 1775)

Thereus cithonius (Godart, [1824])

Thereus praxis (Godman \& Salvin, 1887)

Rekoa meton (Cramer, 1779)

Rekoa palegon (Cramer, 1780)

Rekoa marius (Lucas, 1857)

Arawacus ellida (Hewitson, 1867)

Arawacus aetolus (Sulzer, 1776)

Arawacus tarania (Hewitson, 1868)

Contrafacia imma (Prittwitz, 1865)

Contrafacia sp.

Kolana ligurina (Hewitson, 1874)

Kolana ergina (Hewitson, 1867)

Ocaria ocrisia (Hewitson, 1868)

Chlorostrymon telea Hewitson, 1868

Magnastigma julia Nicolay, 1977

Magnastigma elsa (Hewitson, 1877)

Cyanophrys amynthor (Cramer, 1775)

Cyanophrys herodotus (Fabricius, 1793)

Bistonina mantica (Druce, 1907)

Megathecla cupentus (Stoll, 1871)

Allosmaitia strophius (Godart, [1824])

Lamprospilus badaca (Hewitson, 1868)

Arumecla galliena (Druce, 1912)

Ziegleria hesperitis (Butler \& Druce, 1872)

Ziegleria syllis (Godman \& Salvin, 1877)

Ziegleria perisus (Druce, 1907)

Electrostrymon endymion Fabricius, 1775

Calycopis anthora (Hewitson, 1877)

Calycopis caulonia (Hewitson, 1869)

Calycopis calor (Druce, 1907)

Calycopis demonassa (Hewitson, 1868)

Calycopis calus (Godart, [1824])

Callycopis cissusa (Hewitson, 1877)

Strymon rufofusca (Hewitson, 1867)

Strymon prox. rufofusca sp 1

Strymon prox. rufofusca sp 2

Strymon tegaea (Hewitson, 1868)

Strymon mulucha (Hewitson, 1867)

Strymon crambusa (Hewitson, 1874)

Strymon bazochii (Godart, 1824)

Strymon bubastus (Stoll, 1780)

Strymon eurytulus (Hübner, [1819])

Strymon rana (Schaus, 1902)

Strymon astiocha (Prittwitz, 1865) 
Tabela II Continuação.

$54 \quad$ Strymon cestri (Reakirt, [1867])

55 Strymon ziba (Hewitson, 1868)

56 Strymon prox. ziba

57 Tmolus echion (Linnaeus, 1767)

58 Tmolus venustus (Druce, 1907)

59 Tmolus cydrara (Hewitson, 1868)

$60 \quad$ Nicolaea cauter (Druce, 1907)

$61 \quad$ Nicolaea besidia (Hewitson, 1868)

62 Nicolaea socia (Hewitson, 1868)

63 Nicolaea bagrada (Hewitson, 1868)

$64 \quad$ Nicolaea ophia (Hewitson, 1868)

65 Ministrymon megacles (Stoll, 1780)

66 Ministrymon phrutus (Geyer, 1832)

67 Ministrymon azia (Hewitson, 1873)

68 Ministrymon cleon (Fabricius, 1775)

69 Ministrymon una (Hewitson, 1873)

70 Ministrymon sp.

71 Gargina thyesta (Hewitson, 1869)

72 Gargina gnosia (Hewitson, 1868)

73 Gargina emessa (Hewitson, 1867)

$74 \quad$ Siderus guapila (Schaus, 1913)

75 Siderus philinna (Hewitson, 1868)

76 Theclopsis lydus (Hübner, [1819])

77 Theclopsis gargara (Hewitson,1868)

78 Ostrinotes purpuriticus (Druce, 1907)

79 Ostrinotes keila (Hewitson, 1869)

$80 \quad$ Ostrinotes sophocles (Fabricius, 1793)

81 Strephonota tephraeus (Geyer, 1837)

82 Strephonota jactator (Druce, 1907)

83 Strephonota sphinx (Fabricius, 1775)

$84 \quad$ Strephonota ambrax (Westwood, 1852)

85 Panthiades hebraeus (Hewitson, 1867)

86 Panthiades phaleros (Linnaeus, 1767)

87 Thepytus thyrea (Hewitson, 1867)

88 Oenomaus cortica (D'Abrera, 1995)

89 Parrhasius polibetes (Stoll, 1781)

90 Parrhasius orgia (Hewitson, 1867)

91 Michaelus jebus (Godart, [1824])

92 Michaelus thordesa (Hewitson, 1867)

93 Ignata norax (Druce, 1909)

94 Ignata mulsus (Druce, 1907)

95 Olynthus essus (Herrich-Schäffer, [1853])

96 Aubergina vanessoides (Prittwitz, 1865)

97 Celmia celmus (Cramer, 1775)

98 Dicya carnica (Hewitson, 1873)

99 Chalybs janias Hübner, 1819

100 Symbiopsis lenitas (Druce, 1907)

101 Theclinae sp. 1

102 Theclinae sp. 2

103 Theclinae sp. 3

104 Theclinae sp. 4

105 Theclinae sp. 5

106 Theclinae sp. 6

107 Theclinae sp. 7

108 Theclinae sp. 8

Riodinidae

Euselasiinae - Euselasiini

1 Euselasia pellonia azurea Callaghan, 1999

2 Euselasia zara (Westwood, 1851)

3 Euselasia opalescens (Hewitson, [1855])
Tabela II Continuação.

4 Euselasia melaphaea melaphaea (Hübner, 1823)

5 Euselasia hygenius oculta Stichel, 1919

6 Euselasia mys cytis Stichel, 1919

7 Euselasia thucydides truncata Callaghan, 2001

Riodininae - Mesosemiini

Mesosemia dulcis Stichel, 1910

Mesosemia synnephis Stichel, 1909

Mesosemia melpia vaporosa Stichel, 1910

Mesosemia naiadella dryadela Stichel, 1909

Mesosemia odice (Godart, 1824)

Mesosemia rhodia (Godart, 1824)

Mesosemia bella Sharpe, 1890

Mesosemia cyppus Hewitson, 1859

Mesosemia thera Godman, 1903

10 Mesosemia pardalis Callaghan, 2001

11 Leucochimona icare mathata (Hewitson, 1873)

12 Perophthalma tullius tullius (Fabricius, 1787)

13 Hyphilaria parthenis (Westwood, 1851)

14 Napaea eucharila parvipuncta Lathy, 1932

15 Cremna cuyabaensis Talbot, 1928

16 Cremna thasus thasus (Stoll, 1780)

Riodininae - Eurybiini

1 Eurybia rubeolata planaltensis Callaghan, 2001

2 Eurybia halimede passercula Stichel, 1915

3 Eurybia sp.

$4 \quad$ Alesa prema (Godart, 1824)

Riodininae - Riodinini

Lyropteryx apollonia sparsa Stichel, 1924

Lyropteryx terpsichore terpsichore Westwood, 1851

Cyrenia martia androgyne Stichel, 1910

Ancyluris miniola (Bates, 1868)

Ancyluris meliboeus meliboeus (Fabricius, 1776)

Ancyluris aulestes aulestes (Cramer, 1777)

Ancyluris colubra (Saunders, 1859)

Rhetus periander arthuriana (Sharpe, 1890)

Chorinea licursis (Fabricius, 1775)

10 Chorinea octauius orchestris (Stichel, 1910)

Panara jarbas thymele Stichel, 1909

Brachyglenis drymo (Godman \& Salvin, 1886)

Notheme erota hemicosmeta Seitz, 1917

Monethe alphonsus (Fabricius, 1793)

Chalodeta theodora (C. Felder \& R. Felder, 1862)

Chalodeta chelonis (Hewitson, 1866)

Chalodeta chaonites (Hewitson, 1866)

Dachetola azora (Godart, 1824)

Metacharis lucius (Fabricius, 1793)

Pheles strigosa (Staudinger, 1876)

Pheles atricolor atricolor (Butler, 1871)

Pheles sp.

Barbicornis basilis marginata Seitz, 1913

Syrmatia nyx (Hübner, [1817])

Chamaelimnas tircis pansa Godman, 1903

Chamaelymnas briola meridionalis Lathy, 1932

Detritivora brasilia (Harvey \& Hall, 2002)

Detritivora zama (Bates, 1868)

Detritivora gynaea (Godart, [1824])

Calephelis braziliensis McAlpine, 1971

Parcella amarynthina (C. Felder \& R. Felder, 1865) 
Tabela II Continuação.

$32 \quad$ Baeotis melanis Hübner, [1831]

33 Baeotis johannae johannae Sharpe, 1890

$34 \quad$ Lasaia arsis Staudinger, 1887

35 Lasaia agesilas agesilas (Latreille, 1809)

36 Lasaia pseudomeris Clench, 1972

37 Amarynthis meneria (Cramer, 1776)

38 Riodina lycisca lycisca (Hewitson, 1853)

39 Melanis smithiae smithiae (Westwood, 1851)

$40 \quad$ Melanis aegates lilybaeus (Stichel, 1926)

41 Melanis xenia xenia (Hewitson, [1853])

42 Melanis marathon stenotaenia (Röber, 1904)

Riodininae - Symmachiini

Mesene phareus (Cramer, 1777)

Mesene epaphus epaphus (Stoll,1780)

Mesene monostigma monostigma (Erichson, [1849])

Symmachia probetor probetor (Stoll, 1782)

Symmachia accusatrix Westwood, 1851

Symmachia hippodice Godman, 1903

Symmachia leopardinum Hewitson, 1867

Symmachia almeidai (Zikan, 1946)

Symmachia nemesis Le Cerf, 1958

Pirascca sagaris satnius (Dalman, 1823)

Panaropsis thyatira (Hewitson, 1853)

Phaenochitonia fuliginea (Bates, 1868)

Stichelia bocchoris bocchoris (Hewitson, 1876)

Stichelia cuneifascia (Zikán, 1946)

Riodininae - Helicopini

Sarota gyas (Cramer, 1775)

Anteros formosus formosus (Cramer, 1777)

Anteros lectabilis Stichel, 1909

Anteros principalis Hopffer, 1874

Riodininae - Tribo Incertae Sedis

Emesis condigna Stichel, 1925

Emesis fastidiosa Ménétriés, 1855

Emesis mandana mandana (Cramer, 1780)

Emesis diogenia Prittwitz, 1865

Emesis lupina melancholica Stichel, 1916

Emesis fatimella fatimella Westwood, 1851

Emesis cerea cerea (Linnaeus, 1767)

Emesis temesa peruviana (Lathy, 1904)

Argyrogrammana venilia (Bates, 1868)

Apodemia castanea (Prittwitz, 1865)

Apodemia paucipuncta Spitz, 1930

Riodininae - Nymphidiini

Aricoris constantius (Fabricius, 1793)

Aricoris propitia (Stichel, 1910)

Aricoris hubrichi (Stichel, 1926)

Aricoris aurinia (Hewitson, 1863)

Aricoris colchis (C. Felder \& R. Felder)

Aricoris middletoni (Sharpe, 1890)

Aricoris incana (Stichel, 1910)

Aricoris epulus (Cramer, 1775)

Aricoris caracensis Callaghan, 2001

Ariconias glaphyra (Westwood, 1851)

Lemonias theodora (Godman, 1903)

Lemonias stalachtioides (Butler, 1867)

Thisbe hyalina (Butler, 1867)
Tabela II Continuação.

14 Juditha azan (Westwood, 1851)

15 Juditha molpe (Hübner, [1808])

16 Synargis calyce (C. Felder \& R. Felder, 1862)

17 Synargis gela (Hewitson, [1853])

18 Synargis agle (Hewitson, [1853])

19 Synargis chaonia (Hewitson, [1853])

20 Synargis ethelinda (Hewitson, 1870)

21 Synargis paulistina (Stichel, 1910)

22 Synargis galena (Bates, 1868)

23 Synargis axenus axenus (Hewitson, 1876)

24 Calospila lucianus (Fabricius, 1793)

25 Adelotypa argiella (Bates, 1868)

26 Adelotypa densemaculata (Hewitson, 1870)

27 Adelotypa zerna (Hewitson, 1872)

28 Nymphidium azanoides azanoides Butler, 1867

29 Nymphidium leucosia leucosia (Hübner, [1806])

$30 \quad$ Nymphidium lisimon (Stoll, 1790)

31 Theope eudocia Westwwod, 1851

32 Theope pieridoides C. Felder \& R. Felder, 1865

33 Theope terambus (Godart, [1824])

34 Theope phaeo Prittwitz, 1865

35 Theope thootes (Hewitson, 1860)

36 Theope thestias thestias (Hewitson, 1860)

37 Theope foliorum Bates, 1868

Riodininae - Stalachtini

$1 \quad$ Stalachtis phlegia phlegetontia (Perty, 1833)

2 Stalachtis susanna (Fabricius, 1787)

NYMPHALIDAE

Libytheinae

$1 \quad$ Libytheana carinenta (Cramer, 1779)

Danainae - Danaini

1 Danaus gilippus gilippus (Cramer, 1775)

2 Danaus eresimus plexaure (Godart, 1819)

3 Danaus erippus (Cramer, 1775)

4 Lycorea halia discreta Haensch, 1909

Ithomiinae - Tithoreini

1 Tithorea harmonia pseudethra Butler, 1873

2 Aeria olena Weymer, 1875

3 Aeria elara elarina (Oberthür, 1879)

Ithomiinae - Mechanitini

$1 \quad$ Methona themisto (Hübner, 1818)

2 Thyridia psidii hippodamia (Fabricius, 1775)

3 Mechanitis lysimnia lysimnia (Fabricius, 1793)

4 Mechanitis polymnia casabranca Haensch, 1905

Ithominae - Napeogenini

$1 \quad$ Hypothyris euclea laphria (Doubleday, 1847)

2 Hypothyris ninonia daeta (Boisduval, 1836)

Ithomiinae - Ithomiini

1 Ithomia agnosia agnosia (Hewitson, [1855])

2 Placidina euryanassa (C. Felder \& R. Felder, 1860)

Ithomiinae - Oleriinii

$1 \quad$ Oleria aquata (Weymer, 1875) 
Tabela II Continuação.

Ithomiinae - Dircennini

Dircenna dero dero (Hübner, 1823)

Pteronymia sylvo (Geyer, 1832)

Episcada hymenaea centralis (Brown \& Mielke 1970)

Ithomiinae - Godyridini

Hypoleria lavinia consimilis Talbot, 1928

Hypoleria sarepta goiana d'Almeida, 1951

Brevioleria aelia plisthenes (d'Almeida, 1958)

Brevioleria seba emyra (Haensch, 1905)

Mcclungia cymo salonina (Hewitson, 1855)

Heterosais edessa (Hewitson, [1855])

Pseudoscada erruca (Hewitson, 1855)

Pseudoscada acilla quadrifasciata Talbot, 1928

Morphinae - Morphini

Antirrhea archea Hübner, [1822]

Morpho rhetenor subtusmarina Le Moult \& Réal, 1962

Grasseia menelaus coeruleus (Perry, 1810)

Morpho helenor achillides (C. Felder \& R. Felder, 1867)

Morpho athena Otero, 1966

Morphinae - Brassolini

Narope cyllastros Doubleday, 1849

Narope cyllabarus Westwood, 1851

Narope cyllarus Westwood, 1851

Narope sp. n.

Brassolis sophorae laurentii Stichel, 1925

Brassolis sophorae sophorae (Linnaeus, 1758)

Dynastor darius darius (Fabricius, 1775)

Dasyophthalma rusina rusina (Godart, 1824)

Blepolenis batea ssp. $\mathrm{n}$.

Opsiphanes cassiae crameri C. Felder \& R. Felder, 1862

Opsiphanes invirae remoliatus Fruhstorfer, 1907

Opsiphanes quiteria meridionalis Staudinger, 1887

Selenophanes cassiope (Cramer, 1775)

Catoblepia amphirhoe (Hübner, [1825])

Catoblepia berecynthia (Cramer, 1777)

Eryphanes automedon automedon (Cramer, 1775)

Eryphanes reevesii reevesii (Doubleday, [1849])

Caligo brasiliensis brasiliensis (C. Felder, 1862)

Caligo illioneus illioneus (Cramer, 1775)

Satyrinae - Satyrini

Pampasatyrus ocelloides (Schaus, 1902)

Capronnieria galesus (Godart [1824])

Cercyeuptychia luederwaldti (Spitz, 1931)

Cissia palladia (Butler, 1867)

Cissia penelope (Fabricius, 1775)

Cissia terrestris (Butler, 1866)

Erichthodes narapa (Schaus, 1902)

Euptychia westwoodi Butler, 1867

Fosterinaria stelligera (Butler, 1874)

Godartiana muscosa (Butler, 1870)

Hermeuptychia harmonia (Butler, 1867)

Hermeuptychia hermes (Fabricius, 1775)

Hermeuptychia fallax (C. Felder \& R. Felder, 1862)

Magneuptychia ocnus (Butler, 1867)

Pareuptychia ocirrhoe ocirrhoe (Fabricius, 1777)

Pareuptychia summandosa (Gosse, 1880)

Paryphthimoides numeria (C. Felder \& R. Felder, 1867)
Tabela II Continuação.

18 Paryphthimoides undulata (Butler, 1867)

19 Paryphthimoides phronius (Godart, [1824])

20 Paryphthmoides poltys (Prittwitz, 1865)

21 Pharneuptychia innocentia (C. Felder \& R. Felder, 1867)

22 Pharneuptychia phares (Godart, [1824])

23 Posttaygetis penelea (Cramer, 1777)

24 Praefaunula armilla (Bulter, 1867)

25 Splendeuptychia libitina (Butler, 1870)

26 Splendeuptychia sp.

27 Taygetis chiquitana Forster, 1964

28 Taygetis echo (Cramer, 1775)

29 Taygetis kerea Butler, 1869

$30 \quad$ Taygetis laches (Fabricius, 1793)

31 Taygetis larua C. Felder \& R. Felder, 1867

32 Taygetis mermeria mermeria (Cramer, 1776)

33 Taygetis thamyra (Cramer, 1779)

34 Taygetis tripunctata Weymer, 1907

35 Taygetis virgilia (Cramer, 1776)

36 Taygetomorpha celia (Cramer, 1779)

37 Yphthimoides argyrospila (Butler, 1867)

38 Yphthimoides ochracea (Butler, 1867)

39 Yphthimoides pacta (Weymer, 1911)

$40 \quad$ Yphthimoides straminea (Butler, 1867)

41 Yphthimoides yphthima (C. Felder \& R. Felder, 1867)

42 Yphthimoides celmis (Godart, [1824])

43 Yphthimoides maepius maepius (Godart, [1824])

$44 \quad$ Yphthimoides mythra (Weymer, 1911)

45 Yphthimoides renata (Stoll, 1880)

46 Zischkaia pacarus (Godart, [1824])

Satyrinae - Tribo Incertae sedis

1 Amphidecta calliomma (C. Felder \& R. Felder, 1862)

2 Amphidecta reynoldsi reynoldsi Sharpe, 1890

Charaxinae - Anaeini

Hypna clytemnestra (Cramer, 1777)

Siderone galanthis (Cramer, 1775)

Zaretis sp.

Fountainea glycerium cratias (Hewitson, 1874)

Fountainea ryphea phidile (Geyer, 1837)

Memphis acidalia victoria (Druce, 1877)

Memphis moruus stheno (Prittwitz, 1865)

Charaxinae - Preponini

1 Archaeoprepona amphimachus amphimachus (Fabricius, 1775)

2 Archaeoprepona demophon thalpius (Hübner, [1814])

3 Archaeoprepona demophoon antimache (Hübner, [1819])

4 Archaeoprepona meander meander (Cramer, 1775)

$5 \quad$ Prepona dexamenus dexamenus Hopffer, 1874

6 Prepona laertes demodice (Godart, [1824])

7 Prepona pheridamas (Cramer, 1777)

8 Agrias claudina godmani Fruhstorfer, 1895

Biblidinae - Cyrestini

$1 \quad$ Marpesia chiron (Fabricius, 1775)

2 Marpesia petreus petreus (Cramer, 1776)

Biblidinae - Biblidini

1 Byblis hyperia nectanabis (Fruhstorfer, 1909)

2 Mestra dorcas apicalis (Staudinger, 1886) 
Tabela II Continuação.

3 Catonephele acontius caeruleus (Jenkins, 1985)

4 Catonephele numilia penthia (Hewitson, 1852)

$5 \quad$ Eunica bechina (Hewitson, 1852)

6 Eunica eburnea Fruhstorfer, 1907

$7 \quad$ Eunica macris macris (Godart, 1824)

$8 \quad$ Eunica cuvierii (Godart, 1819)

9 Eunica tatila bellaria Fruhstorfer, 1908

10 Eunica mygdonia mygdonia (Godart, [1824])

11 Eunica volumna volumna (Godart, 1824])

12 Eunica monima (Stoll, 1782)

13 Ectima thecla thecla (Fabricius, 1796)

14 Hamadryas laodamia laodamia (Cramer, 1777)

15 Hamadryas amphinome amphinome (Linnaeus, 1767)

16 Hamadryas februa (Hübner, [1823])

17 Hamadryas feronia feronia (Linnaeus, 1758)

18 Hamadryas iphthime iphthime (Bates, 1864)

19 Hamadryas chloe rhea (Fruhstorfer, 1907)

20 Epiphile orea (Hübner, [1823])

21 Nica flavilla flavilla (Godart, [1824])

22 Temenis laothoe ssp.

23 Temenis huebneri korallion Fruhstorfer, 1912

24 Dynamine postverta postverta (Cramer, 1779)

25 Dynamine tithia tithia (Hübner, [1823])

26 Dynamine artemisia artemisia (Fabricius, 1793)

27 Dynamine agacles agacles (Dalman, 1823)

28 Dynamine athemon (Linnaeus, 1758)

29 Dynamine coenus albidula Weeks, 1901

$30 \quad$ Dynamine limbata (Butler, 1877)

$31 \quad$ Callicore hydaspes (Drury, 1782)

32 Callicore pygas thamyras (Ménétriés, 1857)

33 Callicore astarte selima (Guenée,1872)

34 Callicore sorana sorana (Godart, [1824])

35 Catacore kolyma kolyma (Hewitson, 1852)

36 Diaethria candrena (Godart, [1824])

37 Diaethria clymena janeira (C. Felder, 1862)

38 Diaethria eluina eluina Hewitson, [1855])

39 Haematera pyrame pyrame (Hübner, 1819)

Apaturinae

1 Doxocopa agathina vacuna (Godart, 1824)

2 Doxocopa laurentia (Godart, [1824])

3 Doxocopa linda mileta (Boisduval, 1870)

Nymphalinae - Coeini

$1 \quad$ Colobura dirce dirce (Linnaeus, 1758)

2 Historis acheronta acheronta (Fabricius, 1775)

3 Historis odius dious Lamas, 1995

$4 \quad$ Smyrna blomfildia blomfildia (Fabricius, 1781)

5 Tigridia acesta latifascia (Butler, 1873)

Nymphalinae - Nymphalini

$1 \quad$ Hypanartia lethe (Fabricius, 1793)

2 Vanessa braziliensis (Moore, 1883)

3 Vanessa myrinna (Doubleday, 1849)

Nymphalinae - Kallimini

1 Junonia evarete evarete (Cramer, 1779)

2 Anartia amathea roeselia (Eschscholtz, 1821)

3 Anartia jatrophae jatrophae (Linnaeus, 1763)

$4 \quad$ Siproeta stelenes stelenes (Linnaeus, 1758)

5 Siproeta epaphus trayja Hübner, [1823]
Tabela II Continuação.

\begin{tabular}{|c|c|}
\hline & Nymphalinae - Melitaeini \\
\hline 1 & Chlosyne lacinia saundersi (Doubleday, [1847]) \\
\hline 2 & Anthanassa frisia hermas (Hewitson, 1864) \\
\hline 3 & Eresia eunice esora Hewitson, 1857 \\
\hline 4 & Eresia lansdorfi (Godart, 1819) \\
\hline 5 & Ortilia dicoma (Hewitson, 1864) \\
\hline 6 & Ortilia ithra (Kirby, 1900) \\
\hline 7 & Ortilia sejona (Schaus, 1902) \\
\hline 8 & Phystis simois simois (Hewitson, 1864) \\
\hline 9 & Tegosa claudina (Eschscholtz, 1821) \\
\hline & Limenitidinae - Limenitidini \\
\hline 1 & Adelpha zea (Hewitson, 1850) \\
\hline 2 & Adelpha iphiclus ephesa (Ménétriés, 1857) \\
\hline 3 & Adelpha messana delphicola Fruhstorfer, 1910 \\
\hline 4 & Adelpha capucinus capucinus (Walch, 1775) \\
\hline 5 & Adelpha malea aethalia (C. Felder \& R. Felder, 1867) \\
\hline 6 & Adelpha plesaure phliassa (Godart [1824]) \\
\hline 7 & Adelpha cocala didia (Fruhstorfer, 1915) \\
\hline 8 & Adelpha cytherea aea (C. Felder \& R. Felder, 1867) \\
\hline 9 & Adelpha abia (Hewitson, 1850) \\
\hline 10 & Adelpha calliphane Fruhstorfer, 1915 \\
\hline 11 & Adelpha paraena paraena (Bates, 1865) \\
\hline 12 & Adelpha falcipennis Fruhstorfer, 1915 \\
\hline 13 & Adelpha thoasa gerona (Hewitson, 1867) \\
\hline
\end{tabular}

Heliconiinae - Argynnini

1 Euptoieta hegesia meridiania Stichel, 1938

Heliconiinae - Acraeini

1 Actinote carycina Jordan, 1913

2 Actinote discrepans d'Almeida 1958

3 Actinote melanisans Oberthür, 1917

$4 \quad$ Actinote morio Oberthür, 1917

5 Actinote parapheles Jordan, 1913

6 Actinote pellenea pellenea Hübner, [1821]

7 Actinote rhodope d'Almeida, 1923

8 Actinote surima surima (Schaus, 1902)

Heliconiinae - Heliconiini

1 Agraulis vanillae maculosa (Stichel, [1908])

2 Dione juno suffumata (Brown \& Mielke, 1972)

3 Dryadula phaetusa (Linnaeus, 1758)

4 Dryas iulia alcionea (Cramer, 1779)

$5 \quad$ Eueides isabella dianasa (Hübner, [1806])

$6 \quad$ Eueides aliphera aliphera (Godart, 1819)

7 Heliconius ethilla narcaea Godart, 1819

$8 \quad$ Heliconius besckei Ménétriés, 1857

9 Heliconius erato phyllis (Fabricius, 1775)

10 Heliconius melpomene burchelli Poulton, 1910

11 Heliconius sara thamar (Hübner, [1806])

não garante que determinada espécie esteja de fato protegida. Com o avanço da urbanização e da agricultura, muitas das unidades de conservação vêm sendo transformadas em "ilhas de vegetação", geograficamente isoladas de outras unidades. Os efeitos advindos do isolamento sobre as populações locais, como a interrupção do fluxo gênico, pode ser fatal para a grande 
maioria das espécies, além do estado precário de conservação das unidades (incêndios, caçadores clandestinos, animais domésticos, plantas invasoras, depósitos de lixo e diferentes agentes poluidores são comuns em várias unidades). Outras informações relacionadas à conservação da fauna de borboletas podem ser ainda encontradas em Brown (1991, 1992, 1996).

Agradecimentos. Somos gratos a Olaf H. H. Mielke e Mirna M. Casagrande pela obtenção de dados da Coleção Entomológica Pe. J. S. Moure, Departamento de Zoologia, Universidade Federal do Paraná; André V. L. Freitas, Paulo C. Motta, Ivone R. Diniz, Ronaldo B. Francini, Helena C. de Morais, Amabílio Camargo e Eurides Furtado pela colaboração na identificação de espécies e troca de idéias.

\section{REFERÊNCIAS}

Brown, K. S. Jr. 1991. Conservation of Neotropical environments: insects as indicators, p. 349-404. In: N. M. Collins \& J. A. Thomas (Eds.). The conservation of insects and their habitats. London. Academic Press. 401 p.

Brown, K. S. Jr. 1992. Borboletas da Serra do Japi: diversidade, habitats, recursos alimentares e variação temporal, pp. 142-187. In: L. Morellato (ed.). Historia Natural da Serra do Japi: ecologia e preservação de uma área florestal no sudeste do Brasil. Campinas. Unicamp. 321 p.

Brown, K. S. Jr. 1996. Diversity of Brazilian Lepidoptera: History of study, methods for measurements, and use as indicators for genetic, specific and system richness, p. 221-253. In: C. E. M. Bicudo \& N. A. Menezes (eds). Biodiversity in Brazil: a first approach. São Paulo. CNPq/Instituto de Botânica.

Brown, K. S. Jr. \& A. V. L. Freitas. 2000. Atlantic Forest butterflies: indicators for landscape conservation. Biotropica 32: 934-956.

Brown, K. S. Jr. \& Mielke, O. H. H. 1967a. Lepidoptera of the Central Brazil Plateau. I. Preliminary list of Rhopalocera: Introduction,
Nymphalidae, Libytheidae. Journal of the Lepidopterists Society 21: 77-106

Brown, K. S. Jr. \& Mielke, O. H. H. 1967b. Lepidoptera of the Central Brazil Plateau. II. Preliminary list of Rhopalocera (continued): Lycaenidae, Pieridae, Papilionidae, Hesperiidae. Journal of the Lepidopterists' Society 21: 145-168.

Brown, K. S. Jr., Mielke, O. H. H. \& Casagrande, M. M. 1988. Espécies de Lepidoptera ameaçadas do Estado de Minas Gerais, p. 512-559. In: A. B. M. Machado; G. A. B. Fonseca; R. B. Machado; L. M. S. Aguiar \& L. V. Lins (eds.). Livro vermelho das espécies ameaçadas de extinção da fauna de Minas Gerais. Belo Horizonte, Fundação Biodiversitas. 605 p.

Collins, N. M. \& M. G. Morris. 1985. Threatened Swalowtail Butterflies of the World. The IUCN Red Data Book. Cambridge. $401+$ VIII p.

Diniz, I. R. \& Morais, H. C. 1995. Larvas de Lepidoptera e suas plantas hospedeiras em um cerrado de Brasília, Distrito Federal, Brasil. Revista Brasileira de Entomologia 39: 755-770.

Diniz, I. R. \& Morais, H. C. 1997. Lepidopteran caterpillar fauna of cerrado host plants. Biodiversity and Conservation 6: 817 836.

Eiten, G. 1972. The cerrado vegetation of central Brazil. Botanical Reviews 38: 205-341.

Goodland, R. 1971. A physiognomic analysis of the cerrado vegetation of central Brazil. Journal of Ecology 59: 411-419.

Lamas, G. 2004. Checklist: Part 4A. Hesperioidea - Papilionoidea. In: J. B. Heppner (ed.). Atlas of Neotropical Lepidoptera. Association for Tropical Lepidoptera, Inc. Scientific Publishers. $439 \mathrm{p}$.

Pinheiro, C. E. G. \& J. V. C. Ortiz. 1992. Communities of fruit-feeding butterflies along a vegetation gradient in central Brazil. Journal of Biogeography 19: 505-511.

Pinheiro, C. E. G. 2006. Estudos comparativos sobre a fauna de borboletas do Distrito Federal: Implicações para a conservação. In: A. Scariot; J. C. S. Silva \& J. M. Felfili (eds.). Biodiversidade, Ecologia e Conservação do Cerrado. Brasília, MMA.

Tyler, H.; K. S. Brown Jr. \& K. Wilson. 1994. Swallowtail Butterflies of the Americas: a Study in Biological Dynamics, Ecological Diversity, Biosystematics, and Conservation. Gainesville, Scientific Publishers. 377 p.

Recebido em 17/08/2005; aceito em 13/02/2006 